\title{
THE PREVALENCE AND ANATOMICAL VARIATIONS OF BIFID MANDIBULAR CANAL IN A SAMPLE OF EGYPTIAN POPULATION USING CBCT. A CROSS-SECTIONAL STUDY
}

\author{
Enas M. El Saket *, Salma B. Eiid ** and Ahmad M. Abd El Samad ***
}

\begin{abstract}
Aim: Detection of the prevalence of bifid mandibular canal and classification of its types in the Egyptian population.

Methodology: Radiographic examination of 329 mandibular sides was done using Planmeca Promax 3D Mid CBCT machine. The scans varied depending on patients' sizes (according to the manufacturer's recommendations) using different FOVs with the same voxel size $(0.4 \mathrm{~mm}$ $(400 \mu \mathrm{m})$ ). The bifid the mandibular canal was observed and classified according to Naitoh`s classification (retromolar, dental, forward, and buccolingual canals). In addition, the prevalence of location of accessory canal types and the mean distance to the alveolar ridge and teeth were reported.
\end{abstract}

Results: In 329 hemimandibles, there were 101 bifid canals (30.7\%) and 37 trifid canals $(11.2 \%)$. The dental canal type was the most common (43.3\%), followed by retromolar canal type (33.7\%), forward canal type (17.2\%), and buccolingual canal type (5.7\%). The forward canals and the dental canals had statistically similar mean distances of $11.1 \pm 2.1$ and $11.1 \pm 2.5 \mathrm{~mm}$ respectively. The accessory canals were at a significantly longer mean distance $(12.1 \pm 1.6 \mathrm{~mm})$ to the alveolar crest in the premolar area compared to distance in the molar area $(10.9 \pm 2.3 \mathrm{~mm})$. Retromolar canals showed a significantly longer distance of $13.8 \pm 4.4 \mathrm{~mm}$.

Conclusions: Examination of the mandibular canal is mandatory by surgeons to minimize the chance of missing any accessory branches, since its prevalence was more than $40 \%$ with $21.3 \%$ presented with bilateral manifestation.

KEYWORDS: Mandibular canals, bifid canal, trifid canal, CBCT, CBCT dental.

\footnotetext{
* Former internal resident, Oral and Maxillofacial Radiology Department, Faculty of Dentistry, Cairo University, MSc Degree Candidate, Oral and Maxillofacial Radiology Department, Faculty of Dentistry, Cairo University

** Lecturer, Oral and Maxillofacial Radiology Department, Faculty of Dentistry, Cairo University

*** Associate Professor, Oral and Maxillofacial Radiology Department, Faculty of Dentistry, Cairo University
} 


\section{INTRODUCTION}

The mandibular canal or inferior alveolar canal (IAC) is a hollow tubule spanning the mandible containing a vital structure, called the neurovascular bundle. The identification of such canal becomes mandatory for the planning of a wide range of dental procedures such as insertion of implants, extraction of impacted teeth, surgical planning of biopsies, enucleations of pathologies, and orthognathic surgeries. Normally, the mandibular canal is a single structure, however, it may show some variations in such regard with a single smaller branch or branches sprouting from the main stem called accessory canals. This is labeled bifid and trifid mandibular canals if one or two extra branches were manifested. The observation of bifid mandibular canals was firstly noted in 1973 by Patterson and Funke ${ }^{(27)}$ on panoramic radiographs. Since then, the existence and morphology of bifid mandibular canal were analyzed using various techniques, as panoramic, autopsy, histopathology, computed tomography (CT), and cone beam computed tomography (CBCT). This gave a wide range of prevalence from $0.08 \%$ to $69 \% .^{(1-2-8-21-22-24-25-34)}$

The theory of existence of the bifid and trifid mandibular canals was explained as incomplete fusion of separate mandibular canal nerves from the incisors, primary molars, and permanent molars during embryonic development. ${ }^{(5)}$ Gross anatomical sections and histological examination has confirmed the presence of all or part of the neurovascular bundle. Therefore, according to this diversity in content, the post-operative complications are usually unpredictable ranging from simple to more complicated ones. ${ }^{(10)}$ Irrespective to the content of these canals, they must be adequately examined and described according to their location and direction.

There were several classifications of the bifid mandibular canal that have been reported in literature, which were mainly serving the panoramic examinations. In 1977, Nortje et al ${ }^{(22)}$ classified the bifid canals into three main types of the branches.
Type 1, which is the most common type, where duplicate canals emerge from a single mandibular foramen, whereas type 2 is a short upper canal, which extends to the second or the third molar area. Type 3 is the least common, which emerges as two canals of equal size from two separate foramina that join each other in the molar area. Later, according to anatomical location and configuration,

Langlais et al (1985) (16) have observed and classified the bifid mandibular canal into four types. Type 1 is unilateral or bilateral bifid mandibular canal, which extend to the third molar or the surrounding area. Type 2 is a unilateral or bilateral bifid mandibular canal that run along the course of the main canal and rejoins it at the ramus of the body of the mandible. Type 3 is a combination of the first two ones. Type 4 is described as two canals, originating from a separate mandibular foramen and then join each other to form a larger canal.

Nevertheless, Naitoh et al in $2009^{(21)}$ changed such classifications by observing the origin and direction of branches of the bifid canal on a more advanced imaging technique, the CBCT. Accordingly, it was classified into 4 groups, where forward canal emerges from the superior wall of the main canal, whereas canals emerge from the buccal or the lingual wall are termed bucco-lingual canals. Dental canals are those that end at the root apex of the molars. Lastly, retromolar canal emerges from the main canal turning backwards to the retromolar area.

The significance of existence of these branches is very prominent in several clinical scenarios. In cases of trauma, impingement of the neurovascular bundle usually happens when the fracture is reduced. In the presence of bifid mandibular canal, the alignment becomes more difficult as it is located in another plane. Moreover, in elderly patients as alveolar bone resorbs, the patients with mandibular dentures may experience discomfort if pressure is placed on the neurovascular bundle of the bifid canal. ${ }^{(6)}$ Furthermore, the mandible has been considered as 
an intraoral harvesting donor for autogenous grafts. The most common region for such harvesting is the symphysis and the retromolar region and ignorance of the presence of the retromolar branch will lead to complications. When it comes to daily dental practices, the anesthesia of only lips and chin without the teeth may be an indication of the presence of a bifid canal.

Therefore, it is prudent that assessment of such phenomenon is very crucial and may affect clinicians during their day-to-day procedures. Nevertheless, scarceness of such data was found in Egyptian literature. Therefore, this study was conducted to help clinicians bridge this gap and give better understanding about prevalence and location of mandibular accessory canals.

\section{MATERIALS AND METHODS}

In this observational cross-sectional retrospective study, 329 mandibular sides were analyzed fulfilling the sample size calculation result based on the previous paper by Afsa and Rahmati $2017^{(1)}$, the prevalence of bifid mandibular canal was $31 \%$. Using a precision of 5 , a design effect set at 1 with 95\% CI (confidence interval). The sample size was calculated by Epi info 7 software. Using Planmeca Promax 3D Mid CBCT machine, scans with different FOVs of voxel size $(0.4 \mathrm{~mm}(400 \mu \mathrm{m}))$ were studied using the Planmeca Romexis software viewer version 4.6.2.R (Planmeca). The scans were interpreted by two oral and maxillofacial radiologists (with different experiences) independently and blinded from demographic data of patients and from the results of each other. One radiologist evaluated the images for the presence of bifid canals twice with a time lag of two weeks between the two reading sessions. If secondary canal was present, its configuration (classification) was registered. The second observer evaluated $40 \%$ of the total sample size for inter-reliability assessment. Any disagreement was solved by consensus between the two observers.

\section{Eligibility criteria and selection method:}

Scans of patients aged from 18 to 70 and showing the premolar, molar, retromolar area were included, whereas, scans of patients with mandibular lesions, severe metallic artifacts, previous fractures or surgeries and severe bone resorption till the level of the mandibular canal were excluded from this study.

\section{Image Assessment:}

The primary outcome was the prevalence of bifid mandibular canals in the Egyptian population. Several other outcomes were sought as well such as, the classification of accessory canals, their exact location, and their mean distance in relation to teeth and alveolar crest.

\subsection{Detection of the Bifid Mandibular Canal:}

Before analysis of images, all slice thicknesses and inter-slice distances were adjusted to be 0.4 $\mathrm{mm}$. For proper assessment of the mandibular canal, axial cuts were scrolled to reach a cut with the most adequately displayed mandibular foramen. Reference lines were rotated on the axial image, so that the coronal reference line was perpendicular to the mandibular foramen. This step was repeated also at the mental foramen to assess both areas.

According to the classification of Naitoh et al $2009^{(21)}$, different terms are applied to the extra canal depending on its direction (Figure 1).

1. Forward canal: Emerging from the superior wall of the main canal and extends parallel to the main canal till it fades away.

2. Bucco-lingual canal: Emerging from the buccal or the lingual wall of the main canal.

3. Dental canal: Emerging from the main canal and ends at the root apex of the molars or premolars.

4. Retromolar canal: Emerging from the main canal extending to the retromolar area with/ without an obvious foramen. 


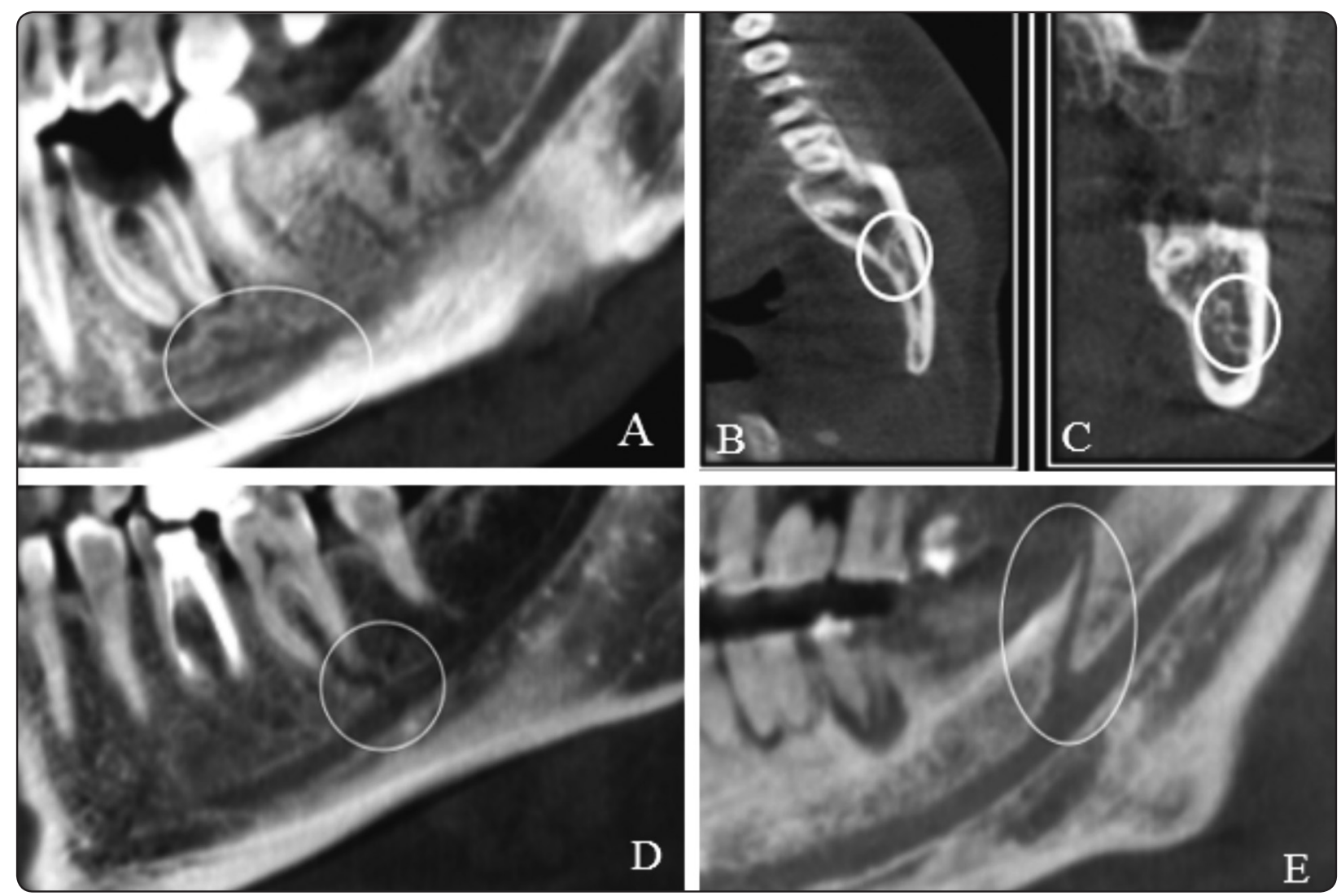

Fig. (1) Different types of mandibular canal branching. (A) Forward canal manifested as a parallel canal of higher level to the main canal in the sagittal cut. Axial CBCT cut showing (B) Buccal canal and coronal CBCT cut showing (C) Lingual canal. Sagittal CBCT cuts showing (D) Dental canal reaching out to the molar apex and (E) Retromolar canal opening superiorly in the retromolar area.

The exit of the retromolar canal was thoroughly checked whether it opens bucally, lingually, superiorly, or just fades away along its path (invisible foramen). The location of buccal/lingual, dental and forward variations were examined whether they were teeth-bearing (premolar/molar teeth/traversing both areas) or ramus-area related (not applied on dental type).

\subsection{Quantitative Assessment:}

Using the built-in measuring tool, distances of forward/dental canals to the alveolar crest and distances of retromolar canals to the mesial of $3^{\text {rd }}$ molars were measured by one observer (EE). First of all, on the axial cut, the sagittal and coronal reference lines were adjusted to be parallel and perpen- dicular to the buccal cortical plate. For retromolar canal measurements, the volume was rotated on corrected sagittal cuts in order to align the occulsal plane to be parallel to the floor. The coronal reference line, which was used as a reference for measurements, was adjusted at the retromolar foramen or the end of the canal. Then, scrolling through sagittal cuts was done to reach the first cut with visible teeth (Figure 2).

Subsequently, the distances from the adjusted coronal reference line to the mesial surface of the 3 rd molar or to the distal surface of the 2 nd molar were measured. For the forward and the dental canals, on the corrected sagittal cut, the alveolar crest was adjusted to be parallel to the floor. Then, scrolling through the sagittal cuts till a clear view 
of the canal was obtained. Thereafter, the axial reference line was adjusted at the end of the detected canal and distances from the end of detected canals to the alveolar crest were measured on the corrected coronal cut.

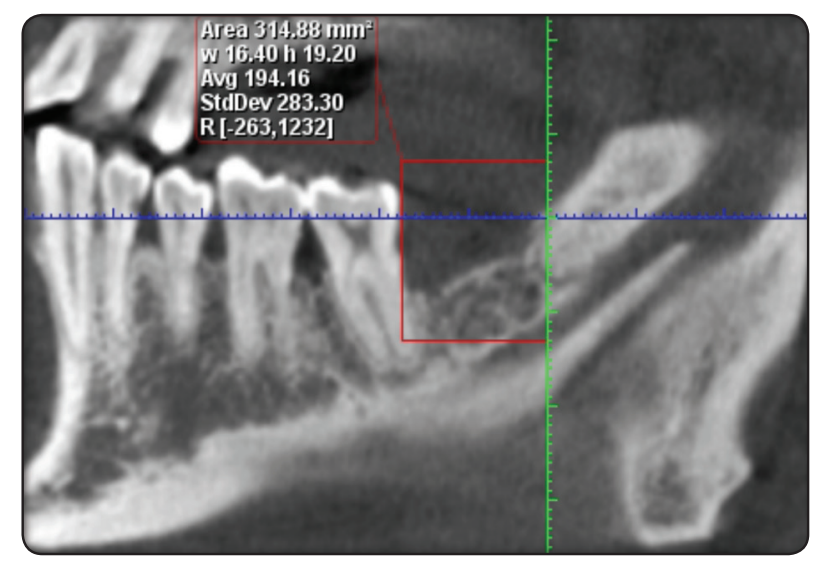

Fig. (2) For the Retromolar canal the distance from the coronal reference line to the tooth was measured to crudely calculate the distance between the retromolar canal and teeth bearing area on the corrected sagittal cut.

\section{Statistical Analysis}

Statistical analysis was performed using IBM SPSS Statistics Version 2.0 for Windows. Data was presented as mean, standard deviation $(\mathrm{SD})$, absolute (n) and relative (\%) frequencies. Kolmogorov-Smirnov and Shapiro-Wilk tests were used to assess data normality of continuous data. The significance level was set at $\mathrm{P} \leq 0.05$. Chisquare test was used to compare prevalence of canal branching and accessory canal types according to gender, lateralization and location. KruskalWallis test followed by Mann-Whitney test were conducted to compare distance of accessory canals from involved teeth/alveolar crest. Intra- and interexaminer reliability regarding canal branching and types of accessory canals was performed using Cohen's Kappa test. Kappa values were interpreted as follows: no agreement $(\leq 0)$, none to slight (0.01-0.20), fair (0.21-0.40), moderate (0.41$0.60)$, substantial $(0.61-0.80)$, and almost perfect agreement (0.81-1).

\section{RESULTS}

In 329 hemimandibles, there were 101 bifid canals $(30.7 \%)$ and 37 trifid canals $(11.2 \%)$ (Table 1). The dental canal type was the most common $(43.3 \%)$, followed by retromolar canal type (33.7\%), forward canal type (17.2\%), and buccolingual canal type $(5.7 \%)$ (Table 2). Thirty-five bilateral canals $(21.3 \%)$ and only one double canal were found. Males showed significantly higher prevalence for the bifid mandibular canal. For lateralization, bifid canals were significantly higher in right sides, whereas trifid canals were abundant in the left side. There was no statistically significant difference in prevalence of types regarding genders and lateralization. The most common location of occurrence of the accessory canals was the molar region (75.4\%,80 cases), followed by the premolar region (20.8\%, 22 cases) and finally the premolar-molar $(1.9 \%, 2$ cases) and ramus (1.9\%, 2 cases) regions.

The forward canals had a mean distance of 11.1 $\pm 2.1 \mathrm{~mm}$, which was statistically similar to mean distance of dental canals that measured $11.1 \pm 2.5$ $\mathrm{mm}$. Retromolar canals showed a significantly longer distance of $13.8 \pm 4.4 \mathrm{~mm}(\mathrm{P}=0.001)$. When assessing each area separately, the accessory canals were at a significantly longer mean distance $(12.1 \pm 1.6 \mathrm{~mm})$ to the alveolar crest in the premolar area compared to distance in the molar area (10.9 $\pm 2.3 \mathrm{~mm})(\mathrm{P}=0.030)$. For the exit of retromolar canals, $54.7 \%$ (29 canals) of the retromolar canals had invisible exits, $43.4 \%$ (23 canals) had superior exits and $1.8 \%$ ( 1 canal) had lingual exit.

Kappa test showed that there was an almost perfect intra-examiner agreement concerning canal branching (Kappa $=0.895, \mathrm{P}=0.000$ ) and accessory canal types (Kappa $=1.000, \mathrm{P}=0.000)$. However, there was a substantial inter-examiner agreement concerning the detection of canal branching (Kap$\mathrm{pa}=0.691, \mathrm{P}=0.000$ ) but almost perfect agreement regarding accessory canal types (Kappa $=0.905$, $\mathrm{P}=0.000$ ). 
TABLE (1): Prevalence (n/\%) of mandibular canal branchings and their prevalence according to gender and side.

\begin{tabular}{|c|c|c|c|c|}
\hline \multicolumn{2}{|c|}{ Total (329) } & Bifid canal & Trifid canal & P value \\
\hline \multicolumn{2}{|c|}{ Frequency (n/\%) } & $101(30.7 \%)$ & $37(11.2 \%)$ & \\
\hline \multirow{2}{*}{ Gender } & Male & $41^{\mathrm{a}}(38.3 \%)$ & $13^{\mathrm{a}}(12.1 \%)$ & \multirow{2}{*}{$0.035^{*}$} \\
\hline & Female & $60^{\mathrm{b}}(27 \%)$ & $24^{\mathrm{a}}(10.8 \%)$ & \\
\hline \multirow{2}{*}{ Side } & Right & $54^{\mathrm{a}}(32.7 \%)$ & $12^{\mathrm{b}}(7.2 \%)$ & \multirow{2}{*}{$0.028 *$} \\
\hline & Left & $47^{\mathrm{b}}(28.6 \%)$ & $25^{\mathrm{a}}(15.2 \%)$ & \\
\hline
\end{tabular}

*: Significant at $P \leq 0.05$

Proportions with different superscript letters within each column are statistically significantly different at P $\leq 0.05$

TABLE (2): Prevalence (n/\%) of accessory canal types and their prevalence according to gender and side

\begin{tabular}{|c|c|c|c|c|c|c|c|}
\hline \multirow{2}{*}{\multicolumn{2}{|c|}{ Total (157) }} & \multirow{2}{*}{ Forward } & \multirow{2}{*}{ Retromolar } & \multirow{2}{*}{ Dental } & \multicolumn{3}{|c|}{ Buccolingual } \\
\hline & & & & & Buccal & & \\
\hline \multirow{2}{*}{\multicolumn{2}{|c|}{ Frequency (n/\%) }} & \multirow{2}{*}{$27(17.2 \%)$} & \multirow{2}{*}{$53(33.7 \%)$} & \multirow{2}{*}{$68(43.3 \%)$} & $6(3.8 \%)$ & \multicolumn{2}{|c|}{$3(1.9 \%)$} \\
\hline & & & & & \multicolumn{3}{|c|}{$9(5.7 \%)$} \\
\hline & & Forward & Retromolar & Dental & Buccolingual & Total & P-value \\
\hline \multirow{2}{*}{ Gender } & Male & $13(20.3 \%)$ & $22(34.3 \%)$ & $27(42.1 \%)$ & $2(3.1 \%)$ & 64 & \multirow{2}{*}{$0.874 \mathrm{NS}$} \\
\hline & Female & $14(15 \%)$ & $31(33.3 \%)$ & $41(44.1 \%)$ & $7(7.5 \%)$ & 93 & \\
\hline \multirow{2}{*}{ Side } & Right & $11(14.8 \%)$ & $24(32.4 \%)$ & $33(44.6 \%)$ & $6(8.1 \%)$ & 74 & \multirow{2}{*}{$0.278 \mathrm{NS}$} \\
\hline & Left & $16(19.2 \%)$ & $29(34.9 \%)$ & $35(42.1 \%)$ & $3(3.6 \%)$ & 83 & \\
\hline
\end{tabular}

NS: non-significant at $P>0.05$

\section{DISCUSSION}

This study comprised a detailed analysis for the bifid and trifid mandibular canals using CBCT, since, it has high resolution, 3-dimensional capabilities and low radiation dose in comparison to $\mathrm{CT}$. For good representation of the prevalence of bifid mandibular canal, a wide age range (18 to 70 years) was selected to represent the Egyptian population. In the scans, reference lines were adjusted twice, once perpendicular at the mandibular canal and the other at the mental foramen, since the pathway of mandibular canal change its direction along the body of the mandible beginning from the mandibular foramen ending to the mental foramen. It's noteworthy that none of the previous reported studies applied this additional step at the mental foramen site. 
In our study and that of Naitoh et al. (2009) ${ }^{(21)}$, Orhan et al. (2011) $)^{(25)}$, Afsa and Rahmati (2017) ${ }^{(1)}$, Luangchana et al. (2019) ${ }^{(19)}$, Okumuş and Dumlu $(2019)^{(23)}$ and DeDeoglu and Duman $(2020)^{(7)}$, the prevalence of bifid canals showed comparable values of $41.9 \%, 43 \%, 46.5 \%, 40.5 \%, 43.6 \%$, $40 \%$ and $41.9 \%$ respectively. However, prevalence showed lower rates in Kuribayashi et al. $\left(2010^{(14)}\right.$, Rashsuren et al. (2014) ${ }^{(29)}$, Kang et al. $\left(2014^{(10)}\right.$, Villaça-Carvalho et al. (2016) ${ }^{(33)}$, Karnasuta et al. (2017) $^{(11)}$, Yang et al. (2017) ${ }^{(34)}$, Laçin et al. (2018) ${ }^{(15)}$, Singh et al. (2018) ${ }^{(31)}$ and Zhou et al. (2020) $)^{(35)}$ studies ranging from $5.63-31.1 \%$.

One important finding in our study was the observation of a high prevalence rate of the trifid mandibular canal of $11.2 \%$ (37 cases). The prevalence of trifid mandibular canal stated by Naitoh et al. (2009) ${ }^{(21)}$, Rashsuren et al. (2014) ${ }^{(29)}$, Yang et al. (2017) $)^{(34)}$, Okumuş and Dumlu (2019 ${ }^{(23)}$ and Zhou et al. (2020) ${ }^{(35)}$ was $3.6 \%, 5.8 \%, 1.1 \%$, $2.4 \%$ and $0.3 \%$ respectively. Furthermore, Afsa and Rahmati $2017^{(1)}$ documented the presence of mandibular canals with 3 and 5 accessory branches of $1.7 \%$ and $0.9 \%$ respectively.

The dental canal was found to be the most commonly occurring type of bifid mandibular canal $(43.3 \%)$. Interestingly enough, and up to our knowledge, none of the authors stated that the dental canals were the most prevalent, where on the contrary, they were the least common type in Orhan et al. (2011) ${ }^{(25)}$ and Orhan et al. (2013) ${ }^{(24)}$ studies of values $4.3 \%$ and $2.4 \%$ respectively. Further sample size calculation was performed to the accessory types and it revealed that only 57 subjects were needed according to Orhan et al. $(2011)^{(25)}$ to extract reliable percentage rates. Thus, this unique dominance of dental canals in our population may show a trustworthy and real distribution.

Regarding gender, in this current study, the prevalence of bifid canal branching was proportionally higher in males than females with statistically significant difference. In line with this finding, the prevalence was higher in males in Kang et al. (2014) $)^{(10)}$, Yang et al. (2017) $)^{(34)}$, Rashsuren et al. (2014) $)^{(29)}$, Zhou et al. (2020) $)^{(35)}$, Singh et al. (2018) $)^{(31)}$ and Laçin et al. (2018) ${ }^{(15)}$ studies but without statistical significance. On the other hand, the prevalence was reported higher in females in Naitoh et al. (2009) $)^{(21)}$, Kuribayashi et al. (2010 ${ }^{(14)}$, Orhan et al. (2011) ${ }^{(25)}$ and Villaça-Carvalho et al. (2016) ${ }^{(33)}$ studies. Regarding lateralization, right sides showed significantly higher prevalence of bifid canals, which corroborated with Laçin et al. $(2018)^{(15)}$ findings.

The highest occurrences of such phenomenon were in the molar region (75.4\%) followed by the premolar region $(20.8 \%)$. This finding was in concordance with Luangchana et al. (2019) (19) who documented the branch prevalence in the molar region to be $80 \%$ and in the premolar region $20 \%$. Some studies reported otherwise, as Afsa and Rahmati (2017) $)^{(1)}$ and Yang et al. (2017) ${ }^{(34)}$ with values of $49.2 \%, 35 \%$ in the molar region and $25.4 \%$, $38 \%$ in the ramus region. Not all studies showed expression of accessory canals in all different areas as Afsa and Rahmati (2017) ${ }^{(1)}$, where they reported that no such occurrence in the premolar region. This can be attributed to the alignment of reference lines to the mandibular foramen only, and being oblivious about the second adjustment to the mental foramen.

A previous knowledge of the exact location of the accessory canals from the alveolar crest is of paramount importance to avoid future neurovascular bundle damage. ${ }^{(35)}$ Zhou et al $(2020)^{(35)}$ measured the end of the accessory branch in relation to the alveolar crest and found that the median vertical distance from all the accessory canal types to the mandibular alveolar ridge was $0.00-8.50 \mathrm{~mm}$. However, in our study, the mean distance for both forward and dental canals exceeded the upper range showing $11.1 \pm 2.1$ and $11.1 \pm 2.5 \mathrm{~mm}$ values and the closest canals to the alveolar crest exhibited distances of 8 and 8.4 $\mathrm{mm}$ respectively. Since the pathway of the main mandibular canal along the jaw differs in molar 
and premolar area, the distance of accessory canals in relation to both was assessed. Accessory canals in the premolar area were at a significantly longer mean distance $(12.1 \pm 1.6 \mathrm{~mm})$ compared to molar teeth $(10.9 \pm 2.3 \mathrm{~mm})$.

Likewise, the distance of the retromolar canal to the teeth was assessed herein, since this area is considered the first choice for the intraoral autologous graft as asserted by $\mathrm{He}$ et al. (2017). ${ }^{(9)}$ Retromolar canals showed a distance of $13.8 \pm 4.4$ $\mathrm{mm}$ to the mesial surface of the $3^{\text {rd }}$ molar. This retromolar finding was corresponding to the studies conducted by Park et al. (2016) ${ }^{(26)}$, Kim et al. (2017) $)^{(12)}$ and Badry et al. $(2020)^{(4)}$, where the mean distance to the distal surface of the second molar was $12.1 \pm 3.3 \mathrm{~mm}, 13.13 \pm 3.24 \mathrm{~mm}$ and $14.70 \pm 5.07 \mathrm{~mm}$ respectively. This will ultimately enrich the Egyptian surgeons with knowledge on estimating safe margins for donor sites when using the $2^{\text {nd }}$ and $3^{\text {rd }}$ molars as references.

In 2007, Misch ${ }^{(20)}$ explained the autologous retromolar graft surgery, stating that the incision begins from retromolar papilla on the midcrest in edentulous patients, where in the dentate patients, the incision begins from the buccal sulcus of retromolar papilla. In addition, another incision is done lateral to the retromolar pad, thus the incisions are done mainly in the superior and buccal sides of the retromolar area. Consequently, it is highly advocated to adequately assess the buccal and superior sides of the retromolar area before planning for autologous graft surgeries in this area. In this study, more than half the cases $(54.7 \%)$ had invisible exits, $43.4 \%$ had superior exits and $1.8 \%$ (one case) had a lingual exit. On the other hand, Sisman et al. $(2015)^{(32)}$ revealed that $88.9 \%$ cases were of visible foramina and classified them into lingual (18.58\%) and buccal (70.36\%) foramina.

In this study, other scenarios have been observed such as bilateral cases having 2 or more branches showing prevalence of $21.3 \%$. It is worthy to note that the accessory canals in bilateral cases were of different types and not identical. Shen et al. (2014) $)^{(30)}$, Yang et al. (2017) $)^{(34)}$, Singh et al. (2018) $)^{(31)}$, Laçin et al. $(2018)^{(15)}$ declared a prevalence of bilateral cases ranging from $2.4 \%-13.9 \%$. In 2010, Kuribayashi et al. ${ }^{(14)}$ were the only CBCT study that reported double canal phenomenon in 2 cases. This manifestation was scarce in reported literature, and in this present study, only one case showed double canals spanning the mandible as two parallel separate canals. On the flip side, higher percentages were usually reported in panoramic-based studies. These false positive bifid canals were due to mimicing the mylohyoid groove to the mandibular canal (Kim et al. 2011). ${ }^{(13)}$ In this current study, multiple cases showed double canals in the sagital cross-sections spanning the mandible parallel to each other. Nevertheless, and thanks to the 3D capabilities of CBCT and assessment using different planes, it showed that these were only grooves in the lingual surface of the mandible.

In 2019, Ramirez ${ }^{(28)}$ published a case report for a unilateral variant of a double canal discovered during the dissection of a 92- year-old African American female. The two distinct mandibular canals showed distinct branches of inferior alveolar artery and mandibular nerve. This finding showcases the threat that might be encountered if these canals are missed during any invasive surgical procedure as well as simpler consequences as inadequate ansthesia. The author even recommended to rely on other cross-sectional techniques such as MRI with better contrast resolution to reveal the contents of these structure before planned surgical procedures. When it comes to risk of injury, it was reported according to Augusto et al., 2015(3) systematic review that the evaluation of the diameters of bifid and trifid accesory branches is considered valuable before surgical procedures.

We can conclude from the current study that careful examination of the course of the mandibular canal is mandatory by Egyptian surgeons to minimize the chance of missing any accessory branches, since 
the prevalence of such event wasn't just occasional especially in the molar region. In addition, it is recommended that large-scale CBCT studies based on sample size calculation should be accomplished for prevalence of bifid canals in subgroups. Additionally, steering the focus on the less common incidents as trifid and double canals, to acquire more valuable recommendations. Furthermore, cohort studies are needed for demonstrating the relation of the location of the retromolar canal and its exit with the site of autologous graft surgery.

\section{REFERENCES}

1. Afsa, M., \& Rahmati, H., (2017). Branching of mandibular canal on cone beam computed tomography images., Singap. Med. J., 38: 21-25.

2. Al-Nakib, L., Ali, J., \& Rashid, S. A., (2011). Identification of bifid mandibular canals among Iraqisubjects using panoramic imaging system. JBCD 23(4): 76-80.

3. Augusto, M., Castro, A., Lagravere-vich, M. O., Amaral, T. M. P., Henrique, M., Abreu, G., \& Mesquita, R. A., (2015). Classifications of mandibular canal branching: A review of literature. World J Radiol, 7(12): 531-538.

4. Badry, M. S. M., El-Badawy, F. M., \& Hamed, W. M., (2020). Incidence of retromolar canal in Egyptian population using CBCT: a retrospective study. EJRNM, 51: 1-8.

5. Chávez-Lomeli, M. E., Mansilla Lory, J., Pompa, J. A., \& Kjaer, I., (1996). The human mandibular canal arises from three separate canals innervating different tooth groups. J. Dent. Res, 75(8): 1540-1544.

6. Claeys, V. and Wackens, G., 2005. Bifid mandibular canal: literature review and case report. DENTOMAXILLOFAC RAD,,34(1), pp.55-58.

7. DeDeoglu, N., \& DUMAN, S. B., (2020). Prevalence of Bifid Mandibular Canals in Turkish Eastern Anatolia Population: A Retrospective Study. J. Clin. Diagnostic Res., 14(2): 13-17.

8. Haghnegahdar, A. A., Bronoosh, P., Khojastepour, L., \& Tahmassebi, P., (2014). Prevalence of bifid mandibular condyle in a selected population in South of Iran. J Dent, 15(4): 156.

9. He, P., Truong, M. K., Adeeb, N., Tubbs, R. S., \& Iwanaga, J., (2017). Clinical anatomy and surgical significance of the lingual foramina and their canals.Clin. Anat., 30(2): 194-204.
10. Kang, J. H., Lee, K. S., Oh, M. G., Choi, H. Y., Lee, S. R., Oh, S. H., Hwang, E. H., (2014). The incidence and configuration of the bifid mandibular canal in Koreans by using cone-beam computed tomography. Imaging Sci Dent, 44(1): 53-60.

11. Karnasuta, P., Plianrungsi, J., Denkongpon, I., Horsimasathaporn, N., Chayutthanabun, P., Weerachartwattana, J., Jutipimarn, K., (2017). Cone-beam computed tomography investigation of crucial mandibular canal variations in Thais. Oral Radiol., 33(3): 219-226.

12. Kim, H.-J., Kang, H., Seo, Y.-S., Kim, D. K., \& Yu, S.-K., (2017). Anatomic evaluation of the retromolar canal by histologic and radiologic analyses. Arch. Oral Biol., 81: 192-197.

13. Kim, M. S., Yoon, S. J., Park, H. W., Kang, J. H., Yang, S. Y., Moon, Y. H., Kim, S. H., (2011). A false presence of bifid mandibular canals in panoramic radiographs. Dentomaxillofac Radiol., 40(7): 434-438.

14. Kuribayashi, A., Watanabe, H., Imaizumi, A., Tantanapornkul, W., Katakami, K., \& Kurabayashi, T., (2010). Bifid mandibular canals: cone beam computed tomography evaluation. DENTOMAXILLOFAC RAD, 39(4): 235-239.

15. Laçin, N., Aytuğar, E., \& Veli, İ., (2018). Cone-beam computed tomography evaluation of bifid mandibular canal in a Turkish population. Int. J. Dent. Res., 8(2): 78-83.

16. Langlais, R. P., Broadus, R., \& Glass, B. J., (1985). Bifid mandibular canals in panoramic radiographs. J Am Dent Assoc, 110(June): 923-926.

17. Littner, M. M., Kaffe, I., Tamse, A., \& Dicapua, P., (1986). Relations

18. hip between the apices of the lower molars and mandibular canal-a radiographic study. Oral Surg Oral Med Oral Pathol Oral Radiol, 62(5): 595-602.

19. Luangchana, P., Pornprasertsuk-Damrongsri, S., Kitisubkanchana, J., \& Wongchuensoontorn, C. (2019). Branching patterns of the inferior alveolar canal in a Thai population: a novel classification using cone beam computed tomography. Quintessence Int., 50(3): 224-231.

20. Misch, C. E., (2007). Contemporary Implant Dentistry, p (993:996), Mosby Elsevier, Philadelphia, PA, USA.

21. Naitoh, M., Hiraiwa, Y., Aimiya, H., \& Ariji, E., (2009). Observation of bifid mandibular canal using cone-beam computerized tomography. INT J ORAL MAX IMPL, 24(1): 155-159.

22. Nortje, C. J., Farman, A. G., \& Grotepass, F. W., (1977). Variations in the normal anatomy of the inferior dental (mandibular) canal: a retrospective study of panoramic 
radiographs from 3612 routine dental patients. Br J Oral Maxillofac Surg 15(1): 55-63.

23. Okumuş, Ö., \& Dumlu, A., (2019). Prevalence of bifid mandibular canal according to gender, type and side. J. Dent. Sci., 14(2): 126-133.

24. Orhan, A. I., Orhan, K., Aksoy, S., Özgül, Ö., Horasan, S., Arslan, A., \& Kocyigit, D. ,(2013). Evaluation of perimandibular neurovascularization with accessory mental foramina using cone-beam computed tomography in children. J. Craniofac. Surg. 24(4): e365-e369.

25. Orhan, K., Aksoy, S., Bilecenoglu, B., Sakul, B. U., \& Paksoy, C. S., (2011). Evaluation of bifid mandibular canals with cone-beam computed tomography in a Turkish adult population: a retrospective study. Surg. Radiol. Anat, 33(6): 501-507.

26. Park, M., Jung, W., Bae, J., \& Kwak, H., (2016). Anatomical and radiographic study of the mandibular retromolar canal. J. Dent. Sci.,11(4): 370-376.

27. Patterson, J.E. and Funke, F.W., 1973. Bifid inferior alveolar canal. Oral Surg Oral Med Oral Pathol Oral Radiol, 36(2), pp.287-288.

28. Ramirez, K. R., (2019). Translational Research in Anatomy Presence of two mandibular canals and distinction of the inferior alveolar and mental nerves proximal to the mandible : A case study. Transl. Res. Anat., 14(January): 6-8.

29. Rashsuren, O., Choi2, J.-W., Han, W.-J., \& Kim, E.-K., (2014). Assessment of bifid and trifid mandibular canals using cone-beam computed tomography. Imaging Sci Dent., 44(3): 229-236.

30. Shen, E.-C., Fu, E., Ming-Jen Fu, M., \& Peng, M., (2014). Configuration and corticalization of the mandibular bifid canal in a Taiwanese adult population: a computed tomography study. INT J ORAL MAX IMPL, 29(4): 894-897.

31. Singh, S., Vineeth, K., Prasad, K., Gupta, V., \& Sonale, S. M. N., (2018). Prevalence of bifid mandibular canal and its relationship with third molar in Indian population: A retrospective analysis. J. adv. clin. res. insights 5(2): 25-29.

32. Sisman, Y., Ercan-Sekerci, A., Payveren-Arıkan, M., \& Sahman, H., (2015). Diagnostic accuracy of cone-beam CT compared with panoramic images in predicting retromolar canal during extraction of impacted mandibular third molars. MED ORAL PATOL ORAL 20(1): e74-e81.

33. Villaça-Carvalho, M. F. L., Manhães, L. R. C., de Moraes, M. E. L., \& de Castro Lopes, S. L. P., (2016). Prevalence of bifid mandibular canals by cone beam computed tomography. Oral Maxillofac Surg, 20(3): 289-294.

34. Yang, X., Lyu, C., \& Zou, D., (2017). Bifid Mandibular Canals Incidence and Anatomical Variations in the Population of Shanghai Area by Cone Beam Computed Tomography. J. Comput. Assist. Tomogr. 41(4): 535-540.

35. Zhou, X., Gao, X., \& Zhang, J., (2020). Bifid mandibular canals: CBCT assessment and macroscopic observation. Surg. Radiol. Anat.: SRA, 1-7. 